\title{
Non-parametric Learning To Aid Path Planning Over Slopes
}

\author{
Sisir Karumanchi, Thomas Allen, Tim Bailey and Steve Scheding \\ ARC Centre of Excellence For Autonomous Systems (CAS), \\ Australian Centre For Field Robotics (ACFR), \\ The University of Sydney, \\ NSW. 2006, Australia. \\ Email: s.karumanchi/t.allen/t.bailey/s.scheding@cas.edu.au
}

\begin{abstract}
This paper addresses the problem of closing the loop from perception to action selection for unmanned ground vehicles, with a focus on navigating slopes. A new non-parametric learning technique is presented to generate a mobility representation where maximum feasible speed is used as a criterion to classify the world. The inputs to the algorithm are terrain gradients derived from an elevation map and past observations of wheel slip. It is argued that such a representation can aid in path planning with improved selection of vehicle heading and operating velocity in off-road slopes. Results of mobility map generation and its benefits to path planning are shown.
\end{abstract}

\section{INTRODUCTION}

Learning techniques that close the loop from perception to action selection are of particular interest for off-road robotics. This loop closure refers to the need for an intermediate module that processes sensed exteroceptive ${ }^{1}$ information into a representation which can directly aid in decision making (such as path planning). In ground vehicle robotics the focus is usually on identifying hard hazards such as obstacles or classifying predefined environmental states into different degrees of traversibility [6], [7], [10], [13]. Assumptions such as terrain homogeneity, or perpetual existence of a road are often made to simplify the problem. In the absence of such assumptions, theoretical techniques that use sensed information to aid decision making need to be investigated. In this paper an intermediate scene interpretation module is proposed in Section II to close the loop from perception to action.

Autonomous navigation in unstructured conditions such as non-homogeneous uneven terrain is a challenging problem to solve. In such environments two main issues need to be addressed. First, explicit assumptions about the terrain should be avoided. Second, in addition to hard hazards (such as obstacles), soft hazards (situations where behaviour needs to be adapted) need to be identified and dealt with. For example, terrain slopes are soft hazards and to successfully negotiate them, vehicle behaviour such as velocity, operating gear and vehicle heading needs to be adjusted.

Due to recent developments in Bayesian non-parametric techniques, learning from experience architectures offer promise. In such architectures, no assumptions need to be

\footnotetext{
${ }^{1}$ Exteroception: perception of external factors that are not under agent control
}

made about the environment. The environment representation is only limited by the available sensor suite and the variables used to define the exteroceptive state. Therefore, experiencebased learning techniques are viable to address the problem of closing the loop from perception to action.

Existing 'learning from experience' techniques include Reinforcement Learning [15] and model predictive techniques [5]. However, they make assumptions such as the existence of a reward function in Reinforcement Learning, or the existence of accurate models for model predictive techniques. It is difficult to quantify such reward functions or develop accurate models in unstructured environments.

Imitation Learning [14] and Inverse Reinforcement Learning [1] are relatively new concepts and have been applied to the problem of learning a reward function from example behaviour. However, they rely heavily on expert input. Controller limitations are usually ignored when systems rely heavily on human input. For example, tuning involved in a PID controller limits the mobility of the platform as it was tuned for a few selected conditions. Such limitations can be dealt with implicitly when the vehicle explores its behavioural capabilities on its own terms.

A learning from proprioception ${ }^{2}$ approach is demonstrated in [2] for a Mars-Rover platform where the authors represent the environment in proprioception space in terms of expected slip. This approach ignores the influence of operating velocity on wheel slip. For a Mars-Rover, proprioceptive measures (such as wheel slip) are mainly dependent on environment conditions and behavioral influences (such as velocity) could be ignored because the platform moves slowly. However, this assumption cannot be made for larger platforms where there is a distribution of slip values for a given condition pertaining to all possible behaviours. Also, such an approach is limited to the case when proprioception is a scalar or a weighted average of scalars. The latter usually involves manual tuning of weights which is not an intuitive process. A single scalar cost cannot capture all the objectives in unstructured conditions. Instead it is beneficial to use a collage of proprioceptive stimuli to judge actions.

\footnotetext{
${ }^{2}$ Proprioception: perception of internal factors that are affected by environment and one's own behaviour.
} 
This paper addresses the problem in question with a specific focus on negotiating two dimensional slopes given range sensor measurements. Section II formally introduces the scene interpretation problem as conditional density estimation and a non-parametric solution using Gaussian Processes is proposed. In Section IV, results are shown on an elevation map derived from laser scans. Mobility maps are derived from the given elevation map by analysing terrain gradients with past observations of vehicle slip collected from a 8x8 skid-steered vehicle (see Figure 1). Finally, in Section V results compare path planning over mobility maps with planning over heuristic costs.

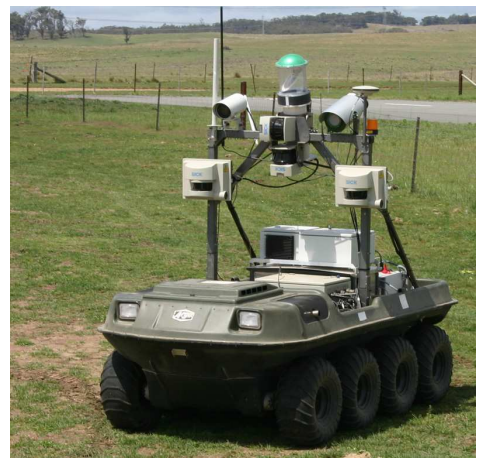

Fig. 1. Argo 8x8 Unmanned Ground Vehicle

\section{PROPRIOCEPTIVE SCENE INTERPRETATION}

\section{A. Motivation}

Current terrain perception modules in unmanned ground vehicles (UGVs) are focused on creating an accurate internal representation of the environment. Exteroceptive parameters such as terrain colour and terrain slope have little value if the vehicle cannot associate them with a value of cost/utility of movement. This task of interpreting exteroceptive data by associating a scalar value of cost or utility is referred to as Scene Interpretation. Better representations in the scene interpretation problem can aid the purpose of bridging the gap between perception and action selection. One cost/utility representation of the world that is of interest is a mobility map [16] (see Figure 2). Here the maximum feasible speed of the vehicle between two points is used as a criterion for continuous classification as such capturing the net utility of an environment condition with a single value. Such a mobility map explicitly represents traversibility of occupied, admissible and unexplored regions and can be used as an objective map for high level trajectory planning algorithms such as $A^{*}$.

For a path planning application, negotiating slopes demands two key requirements in cost representations i) Orientation sensitivity, as navigating down hill and up hill need to be judged differently ii) Ability to encapsulate platform and controller limitations as performance on slopes is very sensitive to controller tuning in practice. Tuning a controller to a certain condition (such as flat terrain) can limit its performance in other conditions (such as non-flat terrain).

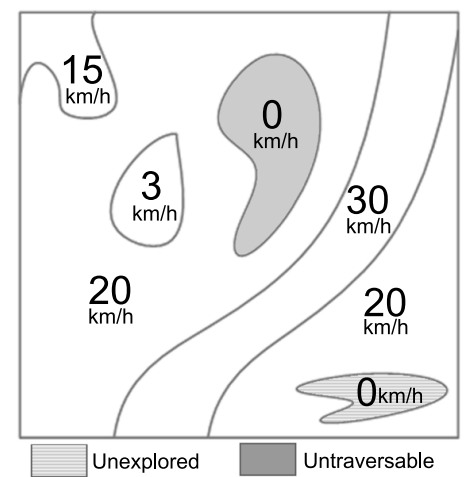

Fig. 2. Sample Mobility Map Indicating Maximum Feasible Speed (Shaded areas indicate immobile and unexplored areas)

A vectorised representation of a mobility map can offer orientation sensitivity by making the mobility values dependent on the direction of pitch and roll slope definitions. An additional benefit of mobility representation over the traditional heuristic cost spaces is that environment utility is defined in behaviour space. In behaviour space, scene interpretation can be treated as a learning problem where agents learn about behavioural limits by physically interacting with the environment. The observed extent of behaviour limitation provides information about environment utility/risk. Both hard and soft hazards are encapsulated in a continuum as different degrees of behavioural limits. Such an interpretation also captures both platform and controller limitations implicitly.

For experience-based scene interpretation, practicality of collecting training data is critical. For example, the learning task of determining behavioural limits can be achieved either in an unsupervised or supervised manner. The former involves optimisation to judge behaviour. Data needs to be collected under all circumstances (including worst case scenarios) to determine optimal behavioural limits. For safety reasons, worst case exploration is not practical on large platforms. Instead, a supervised approach can be developed with an aim to provide an upper bound on feasible actions given access to proprioceptive feedback (wheel slip). Unlike the unsupervised case, data collection is practical as the vehicle only explores what it can negotiate comfortably. Unexplorable behaviour contributes to scene interpretation by indirectly providing information about behaviour limitation.

The need for proprioceptive feedback is to relate exteroceptive states $(\tilde{e})$ such as terrain slopes with action states $(\tilde{a})$ such as vehicle velocity. If a relation can be drawn, a bound on operating velocity can be determined for a given environment condition to create mobility maps. Given no additional information, the two states are independent $(\tilde{e} \perp$ $\tilde{a} \mid \emptyset)$. However, when the right proprioceptive feedback $(\tilde{j})$ is observed, the two become indirectly related $(\tilde{e} \not \perp \tilde{a} \mid \tilde{j})$. This is because of the causal dependencies as both environment and vehicle behaviour affect proprioception and this causes the two sources to be related when the right proprioception is observed. 
The process of analysing and selecting useful proprioceptive measures is a separate problem of its own, and is not dealt with in this paper. For the skid-steered vehicle of interest, slip estimates are chosen as proprioceptive feedback. The slip values cannot be measured directly, so they are estimated with an Unscented Kalman Filter [8] (UKF) using the twotrack process model mentioned in [9]. The reason for using a Kalman filter is to efficiently deal with sensor noise. The test platform has an onboard Inertial Navigation System (INS) and its used to sense vehicle actions such as velocity with good accuracy. In addition, pitch and roll information from the INS are used to sense the current terrain slope (exteroceptive conditions) ${ }^{3}$.

In the next subsections, the notation is summarised in one place to provide easy reference to all the variables and then the theory is introduced.

\section{B. Nomenclature}

$\tilde{x} \quad$ - tilde is used to indicate that a particular variable is a vector.

$\tilde{a}$ - Action vector (operating velocity)

$\tilde{e} \quad$ - Exteroceptive stimuli (terrain slopes)

$\tilde{j} \quad$ - Proprioceptive stimuli (wheel slips): A vector of measures that indicate dependence of performance on environmental conditions and vehicle behaviour.

H - Experience set (training set)

$$
\left(\begin{array}{cccc}
\tilde{e}_{1} & \tilde{e}_{2} & \cdots & \tilde{e}_{N} \\
\tilde{j}_{1} & \tilde{j}_{2} & \cdots & \tilde{j}_{N} \\
\tilde{a}_{1} & \tilde{a}_{2} & \cdots & \tilde{a}_{N}
\end{array}\right)
$$

$\mathbf{J}^{*} \quad$ - Set of proprioceptive stimuli observed in ideal conditions $-\left\{\tilde{j}_{1}^{*}, \tilde{j}_{2}^{*}, \cdots, \tilde{j}_{M}^{*}\right\}$.

$\mathbf{J}^{*}$ is the set of samples derived from a constrained region in proprioception space that is indicative of feasible conditions.

$\mathbf{E}_{\text {test }}$ - Set of test conditions which need to be interpreted (Test set) - $\left\{\tilde{e}_{t e s t 1}, \tilde{e}_{t e s t ~}, \cdots, \tilde{e}_{t e s t T}\right\}$.

for example, the set of both horizontal and vertical gradients for each grid cell form the set of test conditions to interpret terrain slopes from an elevation map.

\section{Problem Definition}

Gathering experience corresponds to collecting co-occurrent observations of $\tilde{e}, \tilde{a}$ and $\tilde{j}$ in as many varied conditions as possible ${ }^{4}$. This experience set $(\mathbf{H})$ serves as a training set for learning. The exploration philosophy for collecting training data is to explore the natural feasibility of vehicle behaviour in

\footnotetext{
${ }^{3}$ The test platform used in this work does not have any suspension, so pitch and roll information from the INS reflects the terrain slope accurately. For other platforms, terrain slopes must be derived from exteroceptive sensors.

${ }^{4}$ Existence of a stationary joint distribution $p(\tilde{e}, \tilde{j}, \tilde{a})$ is assumed. Therefore the experience/training set is a collection of i.i.d samples from the joint.
}

as many varied conditions as possible either under manual or autonomous control. The latter has the advantage of exploring controller limitations.

Before velocity limits can be derived from experience data, an intermediate goal is to infer the feasible behaviour distribution for any test condition given the set of all past observations $(\mathbf{H})$ and the comfortable proprioception set $\left(\mathbf{J}^{*}\right)$ which is chosen by the user to be observations in ideal/nominal conditions. For interpreting slopes, observations from flat terrain conditions are labelled as ideal and used as a reference. This process can be intuitively understood as training the robot what to look for (in proprioception) when exploring feasibility of actions in unknown conditions.

Once feasible behaviour distribution is inferred, an upper bound using the commulative density function (CDF) can determine velocity limits for use in mobility maps. The process of deriving a mobility map given a set of test conditions is outlined below.

Scene Interpretation Process For An Elevation Map

Input: Elevation Map (A set of elevation values)

- Apply the Sobel operator [4] to determine gradient maps in pitch and roll directions (Test set $-\mathbf{E}_{\text {test }}$ ).

foreach $\tilde{e}_{\text {test }}$ in $\mathbf{E}_{\text {test }}$ do
- Infer feasible behaviour distribution from past experience $\left(\mathbf{H} \& \mathbf{J}^{*}\right)$
- Determine operational limit (Maximum Feasible Speed)
end

Output: Mobility Map (Set of all associated mobility values ordered according to their respective test condition in $\mathbf{E}_{\text {test }}$ )

Determining the feasible behaviour distribution is a conditional density estimation problem. The feasible behaviour distribution for a selected environment condition is $p\left(\tilde{a} \mid \tilde{e}_{\text {test }}, i=1\right)^{5}$ where $i$ is an indicator variable to represent the feasibility constraint $\tilde{j} \in \mathbf{J}^{*}$.

$$
i=\left\{\begin{array}{cc}
1 & \tilde{j} \in \mathbf{J}^{*} \\
0 & \tilde{j} \notin \mathbf{J}^{*}
\end{array}\right.
$$

$p\left(\tilde{a} \mid \tilde{e}_{\text {test }}, i=1\right)$ is a measure of confidence in taking an action $\tilde{a}$ given past experience $(\mathbf{H})$. Confidence for an action is based on how often proprioception observed under that action was within the set of proprioceptive stimuli observed in ideal conditions $\left(\mathbf{J}^{*}\right)$ i.e. actions that generated stimuli in the region of accustomed proprioception $\mathbf{J}^{*}$ are preferred.

\section{A Non-Parametric Approximation}

In this section a hierarchical non-parametric ${ }^{6}$ approach is presented to approximate the global conditional density

\footnotetext{
${ }^{5}$ Equivalent to $p\left(\tilde{a} \mid \tilde{e}_{\text {test }}, i=1, \mathbf{H}\right)$ - dependence on the training set $\mathbf{H}$ is not shown for conciseness.

${ }^{6}$ Non-parametric techniques are preferred for learning from experience (memory-based learning) problems as they make the least assumptions about the global form of the distribution.
} 
$p\left(\tilde{a} \mid \tilde{e}_{\text {test }}, i=1\right)$. The local module approximates the function $\tilde{a}=f(\tilde{e}, \tilde{j})$ within a Bayesian non-parametric framework using Gaussian Processes. While the regression module infers local conditional distributions, the global conditional distribution is treated as a kernel density estimation problem where the number of kernels grow as the number of elements in the $\mathbf{J}^{*}$ set grows. Together, the density $p\left(\tilde{a} \mid \tilde{e}_{\text {test }}, i=1, \mathbf{H}\right)$ can be adapted online as the sets $\mathbf{J}^{*}$ and $\mathbf{H}$ grow.

The whole process is captured in the following equation where the desired distribution is derived by marginalising $p(\tilde{a}, \tilde{j} \mid \tilde{e}, i)$ over $\tilde{j}$.

$$
\begin{aligned}
p(\tilde{a} \mid \tilde{e}, i= & =\int p(\tilde{a}, \tilde{j} \mid \tilde{e}, i=1) d \tilde{j} \\
& =\int p(\tilde{a} \mid \tilde{e}, \tilde{j}) p(\tilde{j} \mid i=1) d \tilde{j}
\end{aligned}
$$

$p(\tilde{j} \mid i=1)$ corresponds to the distribution of desired proprioception. In this application, since one has access to $M$ samples of $\tilde{j}$ in the $\mathbf{J}^{*}$ region (i.i.d. samples from $p(\tilde{j} \mid i=1)$ ), the above equation can be approximated as a weighted sum of conditional distributions at the observed $\tilde{j}$ locations.

$$
p(\tilde{a} \mid \tilde{e}, i=1) \approx \sum_{\tilde{j}_{i} \in \mathbf{J}^{*}} \pi_{\tilde{j}_{i}} p\left(\tilde{a} \mid \tilde{e}, \tilde{j}=\tilde{j}_{i}\right)
$$

where $\pi_{\tilde{j}_{i}}$ are mixing components; $\sum \pi_{\tilde{j}_{i}}=1$

If all samples in the $\mathbf{J}^{*}$ set are given equal importance.

$$
p(\tilde{a} \mid \tilde{e}, i=1) \approx \frac{1}{M} \sum_{\tilde{j}_{i} \in \mathbf{J}^{*}} p\left(\tilde{a} \mid \tilde{e}, \tilde{j}=\tilde{j}_{i}\right)
$$

Equation 5 is in the form of kernel density estimation, but with variable kernels, as $p\left(\tilde{a} \mid \tilde{e}=\tilde{e}_{t e s t}, \tilde{j}=\tilde{j}_{i}\right)$ is inferred from data. This can be viewed as an infinite mixture of conditional densities as the number of components grows when the $\mathbf{J}^{*}$ set is allowed to grow. If the local conditionals are approximated to be Gaussian then the global approximation turns out to be a Gaussian mixture.

1) Gaussian Process Regression: Inferring the local conditional distribution $p\left(\tilde{a} \mid \tilde{e}, \tilde{j}=\tilde{j}_{i}\right)$ from observed data at each $\tilde{j}_{i}$ location can be treated as a Bayesian Regression problem $(f:\{\tilde{e}, \tilde{j}\} \rightarrow a)$ [3], [12], where $\tilde{e}, \tilde{j}$ are augmented together to form the input vector $\tilde{x}$ and $a$ is the output $y$.

$$
a_{i}=f\left(\tilde{e}_{i}, \tilde{j}_{i}\right)+\varepsilon
$$

where

$$
\begin{aligned}
& \varepsilon-\text { Noise }-N\left(0, \beta^{-1}\right) \\
& \beta-\text { Noise precision }
\end{aligned}
$$

A Gaussian Process (GP) is completely specified by its covariance function $K\left(x, x^{\prime}\right)$ and its choice defines the space of functions (latent variables - $f$ ) that can be generated [12]. Further, the output is assumed to be zero mean. This is reflected in the prior over the latent variables $p(f)$. Because of this zero mean assumption in GP's , predictions are biased towards null behaviour region (zero) if no data is observed in the test conditions. In the scene interpretation problem, this translates to being cautious in unexplored or underexplored environments which is desired.

$$
\begin{aligned}
P(f)= & N(0, K)-\text { Prior On Functions } \\
& \text { where } \\
& f-\text { latent variables } \\
& K\left(x, x^{\prime}\right)-\text { covariance function } \\
& x=\{\tilde{e}, \tilde{j}\}-\text { input values }
\end{aligned}
$$

The predictive distribution is a Gaussian with the following form:

$$
P\left(a \mid \tilde{e}_{\text {test }}, \tilde{j}_{\text {test }}, \mathbf{H}\right)=N(\mu(x), \operatorname{var}(x))
$$

where

$$
\begin{aligned}
& \mu(x)=K\left(x_{\text {test }}, X\right)\left[K(X, X)+\beta^{-1} I_{N}\right]^{-1} \mathbf{y} \\
& \operatorname{var}(x)=K_{\text {test,test }}+\beta^{-1}-K_{\text {test }}^{T}\left[K+\beta^{-1} I_{N}\right]^{-1} K_{\text {test }} \\
& \mathbf{H}-\left\{a_{\text {train }}, \tilde{e}_{\text {train }}, \tilde{j}_{\text {train }}\right\}_{1 \ldots N}-\text { Training data } \\
& \mathbf{y}-\left\{a_{\text {train }}\right\}_{1 \ldots N}-\text { Training outputs } \\
& X-\left\{\tilde{e}_{\text {train }}, \tilde{j}_{\text {train }}\right\}_{1 \ldots N}-\text { Training inputs } \\
& x_{\text {test }}-\left\{\tilde{e}_{\text {test }}, \tilde{j}_{\text {test }}\right\}-\text { Test input } \\
& K_{\text {test }}=K\left(X, x_{\text {test }}\right) \\
& K_{\text {test,test }}=K\left(x_{\text {test }}, x_{\text {test }}\right)
\end{aligned}
$$

For the scene interpretation problem, the commonly used squared exponential covariance function is chosen. This choice has the stationarity property of associating observations within a local neighbourhood which is desired.

$$
K\left(x, x^{\prime}\right)=\sigma_{f}^{2} \exp \left(-\frac{1}{2 l^{2}}\left(x-x^{\prime}\right)^{2}\right)
$$

The hyper-parameters of the GP $\theta=\left\{l, \sigma_{f}, \beta^{-1}\right\}$ are learned by maximising the log likelihood of the training data (H) using a numerical optimisation technique.

GP regression is a discriminative approach, additional exteroceptive or proprioceptive states could be augmented into the input vector. This allows for incorporating additional sensors or proprioceptive measures into the scene interpretation process. However, inversion of an $N \times N$ matrix $([K(X, X)+$ $\left.\left.\beta^{-1} I_{N}\right]^{-1}\right)$ is its main limitation which is an $O\left(N^{3}\right)$ operation ( $N$ is the size of the dataset). In this work, the inversion was done off-line after the experience data was collected. For online viability, further work needs to be done to investigate techniques that limit the size of the dataset on the fly by either selecting an informative subset within the dataset or dividing the input space with a gating network in a mixture of experts architecture as mentioned in [11]. 


\section{Test Platform, Testing Environment and Data COLLECTION}

The testing platform is a skid-steered vehicle (Figure 1). The platform is equipped with sensors to measure wheel speed, engine RPM, gearbox RPM and brake pressures. It also has an onboard Inertial Navigation System (INS) with access to raw accelerometer and gyro readings from the onboard IMU. The testing environment has access to DGPS (Differential GPS) corrections for the navigation module. The INS system along with GPS/DGPS observations delivers very good localisation (5cm accuracy) and vehicle actions such as velocity are available with good accuracy. Pitch and roll information from the INS are used to sense terrain slope (exteroceptive conditions) so that an elevation map can be interpreted from terrain gradients.

Training data was collected while executing 30 second exploration maneuvers in various terrain conditions. The exploration maneuvers included an acceleration phase, a coasting phase, a turning phase (both left and right turning) and a braking phase to ensure sufficient proprioceptive excitation. The different terrain conditions include flat terrain, uphill, downhill, positive and negative side slope conditions on grass, and a few runs over flat tarmac and a flat gravel road. The exploration runs were repeated for three distinct behaviours (slow: $<1 \mathrm{~m} / \mathrm{s}$, normal: $1-2 \mathrm{~m} / \mathrm{s}$ and fast: $2-3 \mathrm{~m} / \mathrm{s}$ ) on each of the terrain conditions, so as to achieve sufficient exploration in behaviour space. In total, 20 minutes of data was collected at $20 \mathrm{~Hz}$.

\section{ScEne Interpretation Results}

Given training data, and the set of test conditions, the extent of observed movement limitation for each of the test condition needs to be derived. In this section, laser data collected over $100 \times 100 \mathrm{~m}$ off-road terrain is used to derive an elevation map shown in Figure 3 (top). The Sobel operator [4] was applied to the elevation map image to derive pitch and roll gradients, that together form the set of test conditions $\left(\mathbf{E}_{\text {test }}\right)$. Each grid cell has its corresponding exteroceptive state $\left(\tilde{e}_{\text {test }}-\left\{\right.\right.$ slope $_{\text {Pitch }}$, slope $\left.\left._{\text {Roll }}\right\}\right)$ value which needs to be associated with a corresponding velocity limit.

$$
\mathbf{E}_{\text {test }}-\left(\begin{array}{cccc}
\text { slope }_{\text {Pitch } 1} & \text { slope }_{\text {Pitch } 2} & \cdots & \text { slope }_{\text {PitchT }} \\
\text { slope }_{\text {Roll } 1} & \text { slope }_{\text {Roll } 2} & \cdots & \text { slope }_{\text {RollT }}
\end{array}\right)
$$

The slip estimate is two dimensional as observations from the UKF using the two-track process model mentioned in [9] consist of slips observations for both left and right tracks $(\tilde{j}-$ $\left\{\right.$ slip $_{\text {Left }}$, slip $\left.\left._{\text {Right }}\right\}\right)$.

The set of all slip observations obtained from flat terrain conditions are chosen to be the nominal proprioception set $\mathbf{J}^{*}$. Given the desired proprioception set and the experience data from the training runs, a Gaussian Process with a squared exponential covariance function was optimised and the proprioceptive scene interpretation process mentioned in
Section II-C was implemented for the set of slope queries $\left(\mathbf{E}_{\text {test }}\right)$ derived from the gradient maps (see Figure 3).

Each slope condition query results in a Gaussian mixture (see Equation 5). By selecting an upper bound on each of such conditional distribution the maximum feasible speed is determined. The upper bound can be determined from the cumulative density function. Also, a caching data structure is used to prevent interpretation of the same condition twice. This significantly improves the speed of the interpretation process.

The end result of such queries on an elevation map is a mobility map shown in Figure 3. The mobility map interprets the obstacles in the scene (trees) as untraversable with a velocity limit of zero, and the rest of the traversable regions on a continuous scale between $0-7 \mathrm{kmph}$. Brighter the pixel intensity easier it is to traverse.

Mobility is defined in vehicle frame, the direction of movement affects pitch and roll slopes which in turn affects mobility values. In this paper, $A^{*}$ path planning in performed on a grid based representation, hence eight possible directions for slope are considered. Mobility for a given grid cell is a vector of values pertaining to eight possible orientations. Only one such mobility map is shown in Figure 3. All the eight mobility maps are shown in Figure 4. Particularly of interest are maps in Subfigures 4(e) and 4(a), the values for going downhill (4(e)) are significantly smaller than going uphill (4(a)), indicating the need for increased caution.

\section{Path Planning For Slopes}

In Figure 5, path planning over a vector of mobility maps is compared with planning over a scalar cost map. This scalar cost is the maximum gradient of all eight orientations, and the corresponding 'traversibility' map is shown in Figure 4(i).

The key benefit of these mobility maps with respect to planning is that the cost is orientation sensitive. To leverage this benefit in the $A^{*}$ algorithm, the arc cost of a connection between two nodes was given as a function of the particular mobility map associated with the direction of this arc. Figure 5(b) demonstrates the desired sensitivity to platform configuration, whereby the path $\overrightarrow{A B}$, and the reverse path $\overrightarrow{B A}$ take different routes, since the path taken to go downhill is treated differently from going uphill. In the scalar cost map case, shown in Figure 5(a), the paths $\overrightarrow{A B}$ and $\overrightarrow{B A}$ are the same.

The $A^{*}$ paths in Figure 5 only offer heading commands and no information about velocity, so the heuristic cost path in Figure 5(a) needs to be operated with a constant speed preselected for cautious navigation (usually about $1 \mathrm{~m} / \mathrm{s}$ or $3.6 \mathrm{~km} / \mathrm{hr}$ for the platform in question). For the second case, information from the eight mobility maps can be used to regulate or bound velocities. Average 'maximum feasible speeds' for the paths in Figure 5(b) are $6.1121 \mathrm{~km} / \mathrm{hr}$ in the forward path (white) and $5.9079 \mathrm{~km} / \mathrm{hr}$ in the backward path (green). These values are an improvement from that of the cautious case as the mobility values adjust to situations of caution by slowing down and situations of confidence by speeding up. 


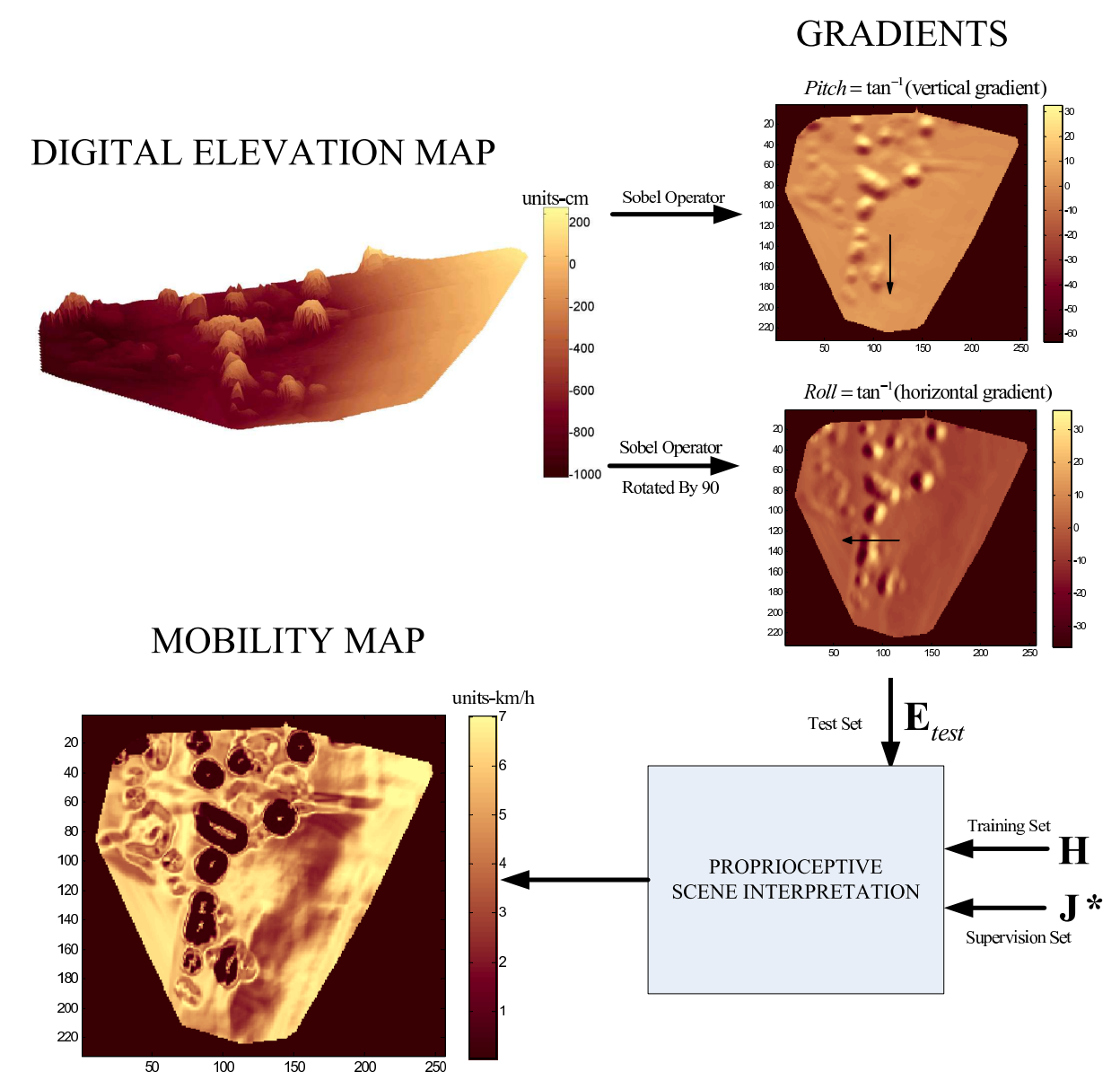

Fig. 3. Scene interpretation of an elevation map $(\sim 100 \times 120 \mathrm{~m}$ at $0.5 \mathrm{~m}$ grid resolution) derived from laser data (units of mobility $=k m / h r)$. The mobility map shown assumes that the vehicle intends to travels in the downwards direction shown in the pitch gradient map.

In a separate experiment, controller performance is compared for paths planned on a $5^{\circ}$ hill (see Figure 6(a)) with and without mobility paths. For a given set of four waypoints, $A^{*}$ was used to plan across the heuristic scalar cost representation and across mobility maps. The path given by the scalar frame work is executed for constant values of velocity starting from $1 \mathrm{~m} / \mathrm{s}$ to $3 \mathrm{~m} / \mathrm{s}(3.6-10.8 \mathrm{~km} / \mathrm{h})$. In contrast the second path planned over mobility maps uses values from the maps to regulate velocity. Figures 6(b) and 6(c) show the input waypoints, the planned paths and the executed paths.

For this experiment, training data were collected under autonomous control where the tuned controller is part of the system. The control system was tuned on flat grass conditions, hence it does not perform well in non-flat conditions (especially downhill where the system becomes significantly underdamped for higher velocities). By performing the same scene interpretation process as before with this new training data the controller limitations are captured into the mobility representation. In Table I the paths are compared for average speed and tracking performance. Tracking performance is judged by standard control theoretic metrics such as root mean squared error (RMSE), $L_{\infty}$ norm (max error value) and the
$L_{2}$ norm. It can be seen that the path over mobility maps shows improved controller performance for similar average and maximum speeds as the path with the highest constant velocity of $3 \mathrm{~m} / \mathrm{s}$. This improvement is due to the vehicle slowing down in downhill conditions and speeding up on flat terrain.

While the results in Figure 5 illustrate the desired orientation sensitivity in paths, results in Table I show that controller limitations are captured in the mobility representation and help in regulating velocity to improve controllability. These results illustrate that accounting for directional mobility are of benefit for UGV planning. Use of mobility information leads to a safer choice of paths with a reduced risk of excessive slippage and improved controllability as the system automatically decides on the feasible velocity of operation.

\section{CONCLUSION AND Discussion}

This paper presents a non-parametric learning technique to interpret terrain slopes from wheel-slip observations, and describes the benefits of defining costs in behaviour space (as operational limits). First, this enables experience-based learning and encapsulates platform and controller limitations in a common representation. Second, behaviour dependent 


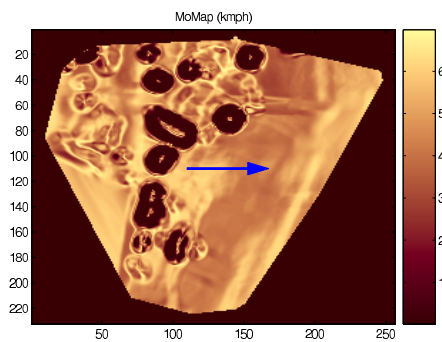

(a) Uphill

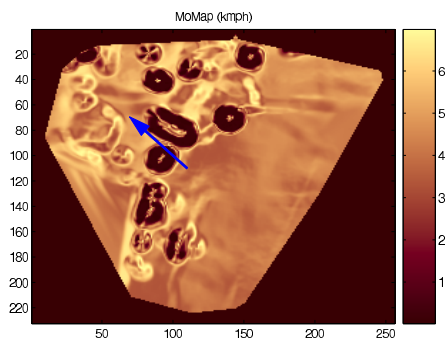

(d)

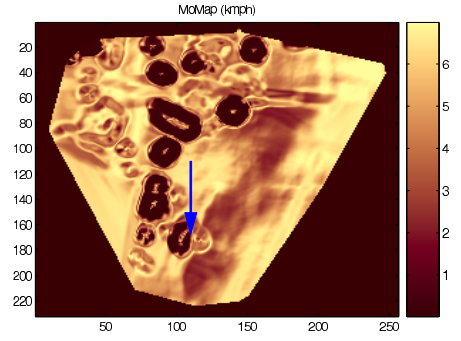

(g)

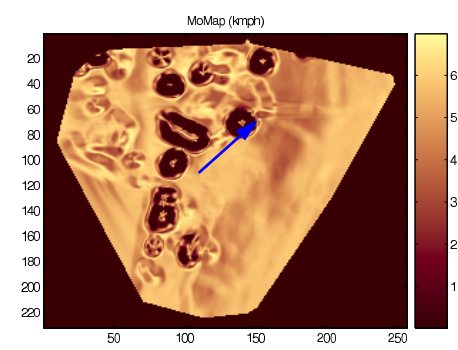

(b)

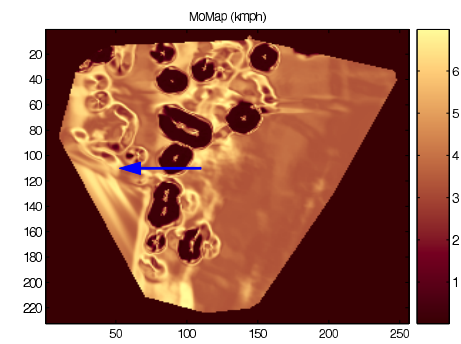

(e) Downhill

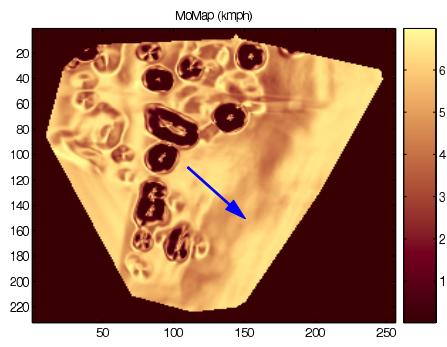

(h)

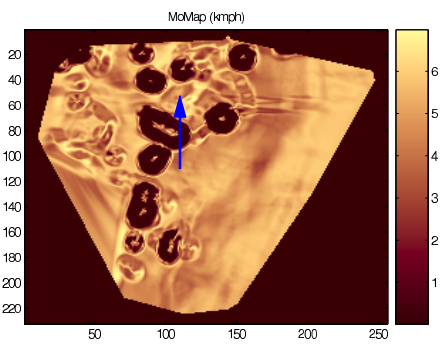

(c)

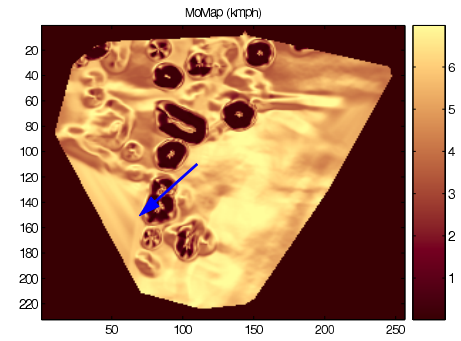

(f)

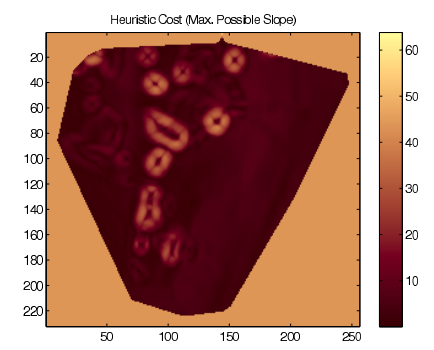

(i) A scalar heuristic cost: max. possible slope (brighter pixels have higher cost; notice that unknown regions have high cost)

Fig. 4. Mobility Maps for eight possible vehicle headings (a-h) and an alternative scalar cost representation (i)
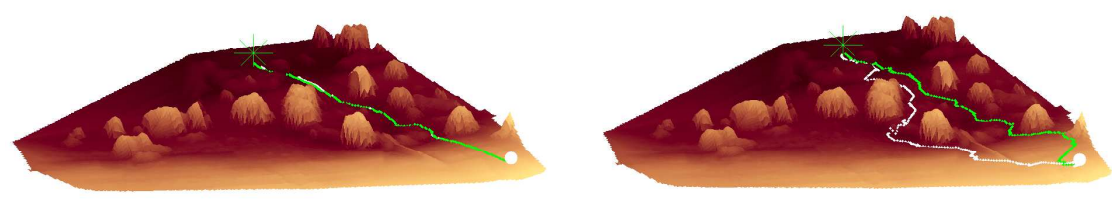

(a) $A^{*}$ path planning on heuristic cost map (path (b) $A^{*}$ path planning on directional mobility maps distance: $101 \mathrm{~m}$ ) (path distance: $121 \mathrm{~m}$ forward and $109 \mathrm{~m}$ backward)

Fig. 5. Path planning on heuristic cost maps vs. directional mobility maps. [white $\bullet$ (start $) \rightarrow$ white path $\rightarrow$ green $*$ (goal) $\rightarrow$ green path $\rightarrow$ start]

TABLE I

TRAJECTORY FOLLOWING RESULTS: AVERAGE SPEED AND TRACKING PERFORMANCE

\begin{tabular}{|c|rrr|rrr|}
\hline \hline & \multicolumn{3}{|c|}{ Speed performance } & \multicolumn{3}{|c|}{ Cross track error performance } \\
& time $(s)$ & Mean Vel $(m / s)$ & Max. Vel $(m / s)$ & RMSE & $L_{\infty}$ norm & \multicolumn{1}{c|}{$L_{2}$ norm } \\
\hline$A^{*}+1 m / s$ & 130 & 0.95 & 1.70 & 1.15 & 2.34 & 158.65 \\
$A^{*}+2 m / s$ & 73 & 1.71 & 2.62 & 1.61 & 3.66 & 254.56 \\
$A^{*}+3 m / s$ & 65 & 1.99 & 3.41 & 2.14 & 5.69 & 536.04 \\
$A^{*}+$ Mobility Maps & 64 & 2.07 & 3.29 & 1.98 & 4.54 & 360.03 \\
\hline
\end{tabular}




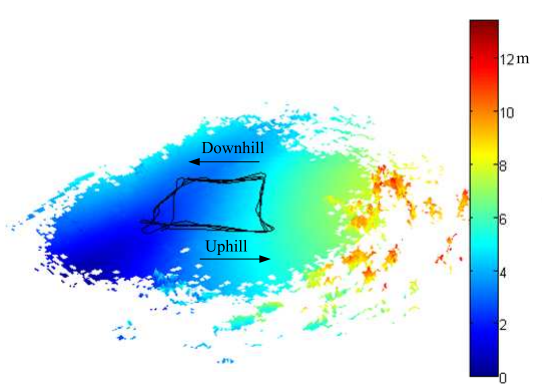

(a) Trajectory test environment - A $5^{\circ}$ Hill

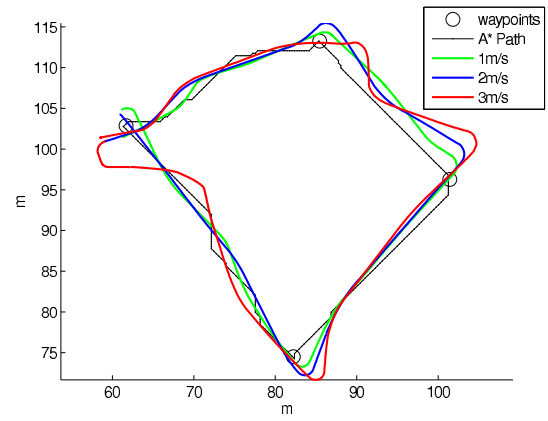

(b) $A^{*}$ Path on scalar heuristic cost (black) and the executed trajectories with different constant velocities (green-1m/s,blue- $2 \mathrm{~m} / \mathrm{s}$,red- $3 \mathrm{~m} / \mathrm{s}$ )

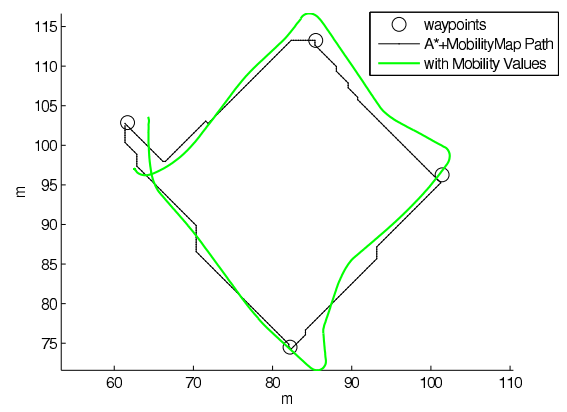

(c) $A^{*}$ Path on mobility maps (black) and the executed trajectory with mobility values (green)

Fig. 6. Trajectory following experiment

costs can be created for aiding decision making, such as orientation sensitive costs for UGV path planning. Finally, proprioceptive feedback such as wheel slip can be incorporated and used to learn effectively in complex environments.

The current process of creating the mobility map is slow, as it involves inverting an $N \times N$ matrix (where $N$ is the size of the dataset). In this work, the inversion was done offline, and the size of dataset was of the order of 10000 data points. The mobility maps shown took about 15 minutes to generate on a $2 \mathrm{GHz} \mathrm{PC}$ in a Matlab implementation. After the first interpretation, the inverted matrix and the interpreted values were cached and the subsequent queries were very quick in comparison. A new query for an unknown test condition took approximately 0.3 seconds, but cached queries took approximately 0.03 seconds. Depending on the number of repeated test conditions, the speed of the process will vary for a given scene.

Non-parametric techniques and experience based learning, although not viable as a full online process at present, are a sensible approach in off-road unstructured conditions where simplifying assumptions about the environment cannot be made. They are practical for off-line analysis of sensor information to be used as prior data for path planning. More importantly, they provide a theoretical approach to the process of defining and generating costs. Sparsification and local approximations in GP's is an active area of research, and these techniques can be used in the future to make it more viable.

\section{ACKNOWLEDGMENT}

This work is supported by the ARC Centre of Excellence programme, funded by the Australian Research Council (ARC) and the New South Wales State Government.

\section{REFERENCES}

[1] Pieter Abbeel and Andrew Y. Ng. Apprenticeship learning via inverse reinforcement learning. In 21st International Conference on Machine Learning, Banff, Canada, 2004

[2] Anelia Angelova, L. Matthies, D. Helmick, and P. Perona. Learning and prediction of slip using visual information. Journal of Field Robotics, 2007.

[3] C. M. Bishop. Pattern Recognition And Machine Learning. Springer, 2006.

[4] Rafael C. Gonzalez and Richard E. Woods. Digital Image Processing. Prentice Hall, 3rd edition edition, 2008.

[5] Alexander R. Green and David Rye. Sensible planning for vehicles operating over difficult unstructured terrains. In IEEE Aerospace Conference, pages 1-8, 2007.

[6] R. Hadsell, P. Sermanet, A. N. Erkan, J. Ben, J. Han, B. Flepp, U. Muller, and Y. LeCun. Online learning for offroad robots: Using spatial label propagation to learn by long-range traversability. Proceedings of Robotics: Science and Systems, 2007.

[7] L. D. Jackel, Eric Krotkov, Michael Perschbacher, Jim Pippine, and Chad Sullivan. The darpa lagr program: Goals, challenges, methodology, and phase i results. Journal of Field Robotics, 23(11-12):945-973, 2006.

[8] S. J. Julier, J. K. Uhlmann, and H. F. Durrant-Whyte. A new method for the nonlinear transformation of means and covariances in filters and estimators. In IEEE Transactions on Automatic Control, volume 45, 2000.

[9] Anh Tuan Le, D.C. Rye, and H.F. Durrant-Whyte. Estimation of track-soil interactions for autonomous tracked vehicles. Robotics and Automation, 1997. Proceedings., 1997 IEEE International Conference on, 2:1388-1393 vol.2, Apr 1997.

[10] L. Ojeda, J. Borenstein, G. Witus, and R. Karlsen. Terrain characterization and classification with a mobile robot. Journal Of Field Robotics, 2006.

[11] C. E. Rasmussen and Z. Ghahramani. Infinite mixtures of gaussian process experts. In In Advances in Neural Information Processing Systems 14, pages 881-888. MIT Press, 2002.

[12] C. E. Rasmussen and C. K. I. Williams. Gaussian Processes for Machine Learning. MIT Press, 2006.

[13] Michael Shneier, Tommy Chang, Tsai Hong, Will Shackleford, Roger Bostelman, and James S. Albus. Learning traversability models for autonomous mobile vehicles. Autonomous Robots, 24(1), 2008.

[14] David Silver, James Bagnell, and Anthony Stentz. High performance outdoor navigation from overhead data using imitation learning. In Robotics: Science and Systems IV, Zurich, Switzerland, 2008.

[15] R. S. Sutton and A. G. Barto. Reinforcement Learning: An Introduction. The MIT Press, 1998.

[16] J. Y. Wong. Theory Of Ground Vehicles. New York: Wiley, 3rd edition edition, 2001. 